\title{
BMJ open The impact of targeting all elderly persons in England and Wales for yearly influenza vaccination: excess mortality due to pneumonia or influenza and time trend study
}

\author{
Andrea G Mann, ${ }^{1}$ Punam Mangtani, ${ }^{1}$ Colin A Russell, ${ }^{2,3}$ John C Whittaker ${ }^{4,5}$
}

To cite: Mann AG, Mangtani P, Russell CA, et al. The impact of targeting all elderly persons in England and Wales for yearly influenza vaccination: excess mortality due to pneumonia or influenza and time trend study. BMJ Open 2013;3: e002743. doi:10.1136/ bmjopen-2013-002743

- Prepublication history and additional material for this paper is available online. To view these files please visit the journal online (http://dx.doi.org/10.1136/ bmjopen-2013-002743)

Received 17 February 2013 Revised 1 May 2013 Accepted 16 May 2013

For numbered affiliations see end of article.

\section{Correspondence to} Dr Andrea G Mann; andrea.mann@Ishtm.ac.uk

\section{ABSTRACT}

Objective: To investigate the impact on mortality due to pneumonia or influenza of the change from riskbased to age group-based targeting of the elderly for yearly influenza vaccination in England and Wales.

Design: Excess mortality estimated using time series of deaths registered to pneumonia or influenza, accounting for seasonality, trend and artefacts. Nonexcess mortality plotted as proxy for long-term trend in mortality.

Setting: England and Wales.

Participants: Persons aged 65-74 and 75+ years whose deaths were registered to underlying pneumonia or influenza between 1975/1976 and 2004/2005.

Outcome measures: Multiplicative effect on average excess pneumonia and influenza deaths each winter in the 4-6 winters since age group-based targeting of vaccination was introduced (in persons aged $75+$ years from 1998/1999; in persons aged 65+ years from 2000/2001), estimated using multivariable regression adjusted for temperature, antigenic drift and vaccine mismatch, and stratified by dominant circulating influenza subtype. Trend in baseline weekly pneumonia and influenza death rates.

Results: There is a suggestion of lower average excess mortality in the six winters after age groupbased targeting began compared to before, but the $\mathrm{Cl}$ for the 65-74 years age group includes no difference. Trend in baseline pneumonia and influenza mortality shows an apparent downward turning point around 2000 for the 65-74 years age group and from the mid-1990s in the $75+$ years age group.

Conclusions: There is weakly supportive evidence that the marked increases in vaccine coverage accompanying the switch from risk-based to age group-based targeting of the elderly for yearly influenza vaccination in England and Wales were associated with lower levels of pneumonia and influenza mortality in older people in the first 6 years after age group-based targeting began. The possible impact of these policy changes is observed as weak evidence for lower average excess mortality as well as a turning point in baseline mortality coincident with the changes.

\section{ARTICLE SUMMARY}

\section{Article focus}

- There has been no assessment of the impact on mortality of the switch from risk-based to age group-based targeting of yearly influenza vaccination of the elderly in England and Wales which was phased in from 1998/1999 and which resulted in a marked increase in yearly vaccine coverage.

- Our aim was to investigate the impact on mortality due to pneumonia or influenza of the change from risk-based to age group-based targeting of the elderly for yearly influenza vaccination in England and Wales.

\section{Key message}

- Our study provides weak evidence for lower influenza-related mortality under age groupbased targeting compared with risk-based targeting of yearly influenza vaccination of the elderly.

Strengths and limitations of this study

- Strengths are that we analysed a long time series of data, carefully controlling for changes in coding and laboratory practices and using the most specific mortality outcome available.

- Limitations include potential underestimation of mortality and residual confounding.

\section{INTRODUCTION}

There has been no assessment of the impact on mortality of the switch from risk-based to age group-based targeting of yearly influenza vaccination of the elderly in England and Wales. The policy of targeting people for yearly influenza vaccination based on risk alone, in place until 1998/1999, meant that only people with certain medical conditions (chronic heart, respiratory or renal disease, diabetes mellitus or immunosuppression) were offered free yearly influenza vaccination. ${ }^{1}$ The age group-based targeting policies 
adopted in 1998-2000 meant that from then on, all those people aged 75 years and over (from 1998) and aged 65 years and over (from 2000) would be offered free yearly influenza vaccination. These policy changes were followed by a marked increase in vaccination coverage of those aged 65 years and over, from $46 \%$ in the winter of $1999 / 2000$ to $66 \%$ in $2000 / 2001$ (calculated based on reported coverage in high-risk and low-risk people aged $65+$ years in [2]). Vaccine coverage has continued to rise or stay above this level ever since. ${ }^{2-4}$ Evidence for lower excess mortality due to pneumonia or influenza (P\&I) in the elderly in the years shortly following the move to age group-based targeting, or for a fall in baseline respiratory mortality in the elderly coincident with these policy changes, would be evidence that these changes have prevented more elderly deaths than the former risk-based approach to vaccine targeting.

Debate surrounds the question of the efficacy and effectiveness of influenza vaccine in the elderly. ${ }^{5-8}$ Two systematic reviews concluded that there is insufficient good quality evidence, ${ }^{9} 10$ though one of these reviews did include a randomised controlled trial showing efficacy against influenza disease of $58 \%$ (95\% CI $26 \%$ to $77 \%$ ) in adults aged 60 years and over. ${ }^{11}$ Observational studies controlling for positive confounding by healthier people seeking vaccination have also demonstrated effectiveness against mortality. ${ }^{12} 13$ There has been much interest in measuring the mortality impact of influenza vaccination of the elderly in other settings and the results have been mixed. In Holland, all-cause excess mortality during influenza epidemics declined after the introduction of universal yearly vaccination of those aged 65 years and over. ${ }^{14}$ In contrast, three other studies, in the USA, Italy and Catalonia, did not detect an impact of rising vaccine coverage of the elderly on all-cause excess mortality. ${ }^{15-17}$ Previous studies of influenza attributable or excess mortality in the UK have not related this to vaccination. ${ }^{18-20}$

While the level of mortality observed when influenza is circulating in the community (often quantified using excess mortality) varies from winter to winter, baseline respiratory mortality (ie, excluding excess) has a seasonal pattern which is more or less constant from winter to winter, though it may change over the long term. ${ }^{21}$ Previous studies of the long-term trends in influenza-related mortality in England and Wales have covered earlier, and usually shorter, time periods. One study examined trends in P\&I mortality in England and Wales from 1994/1995 to 2000/2001 and showed a plot of rates of P\&I mortality in the 65-74 years age group in this period, which appears to have little secular trend. ${ }^{18}$ The shape of the trend in baseline respiratory mortality in England and Wales since 2000/2001 is unclear.

This work evaluates a public health initiative to specifically target all people aged 65 years and older for yearly influenza vaccination regardless of their risk group, which has been in place since 2000. Studying patterns in excess mortality and trends in baseline mortality in the years shortly following the introduction of this initiative allows us to provide evidence for the impact of this policy.

\section{METHODS}

Daily counts of deaths registered to underlying P\&I in England and Wales between 1975 and 2005, by date of death, sex and age group, were provided by the Office for National Statistics (ONS). Deaths registered to underlying P\&I, not just confirmed influenza deaths, were analysed because deaths from influenza are rarely laboratory confirmed and because deaths in the elderly, which occur secondary to influenza, often result from pneumonia. ${ }^{22} 23$ P\&I mortality rates are a more specific measure of influenza activity than rates of all-cause mortality. ${ }^{24}$

The mortality data were adjusted by multiplying them by conversion factors estimated in a separate analysis to account for the following historical modifications to how the data are coded so that our assessment of trends would not be influenced by these artefactual step changes. The International Classification of Diseases (ICD) changed from V.8 to V.9 in 1979, leading to a small decline in deaths coded to underlying P\&I. ${ }^{25}$ In 1984, ONS introduced a broader interpretation of rule 3 for coding the underlying cause of death, which led to an abrupt fall in deaths registered to underlying pneumonia, and to a rise in deaths registered to underlying cancer and ischaemic heart disease. ${ }^{26}$ In 1993, ONS adopted an automated system for coding the underlying cause of death, which narrowed the interpretation of rule 3 and approximately reversed the change adopted in 1984 (ie, rates of deaths being registered to underlying pneumonia rose back to a level approximately equal to that pre-1984). ${ }^{27}$ With the change from ICD 9 to ICD 10 in 2000 , deaths coded to underlying respiratory disease fell by approximately $22 \%$, and deaths coded specifically to underlying pneumonia fell by $38 \% .^{28}$

The Health Protection Agency Centre for Infections (HPA CfI) provided an extract of all individual reports of laboratory-confirmed influenza A infections between 1975 and 2005 from their LabBase2 database. These reports, based on virus isolation and PCR, were reported voluntarily by the National Health Service (NHS) and HPA laboratories in England and Wales. Records included individuals' age, sex and the earliest specimen date.

The statistical methods used are summarised below, with full details provided in online supplementary material. Vaccine coverage estimates for 1989/1990-2004/2005 were adapted from published sources. Published estimates of vaccine coverage were available by age group and separately for persons considered at high or low risk of influenza complications. ${ }^{2}{ }^{3}$ Separate estimates by risk group were combined proportionately according to the number of each group vaccinated to give coverage for that age group regardless of the risk group. 
There are numerous approaches in the literature to quantifying excess mortality as a measure of influenza severity but no gold standard approach. We elected to modify the method of Simonsen et al, ${ }^{15}$ used in their paper analysing influenza vaccine impact in the USA, to examine excess mortality as well as the long-term trend in non-excess mortality in relation to changes in vaccine policy and coverage. The Simonsen method is a modification of Serfling's cyclical regression approach, ${ }^{21}$ where non-epidemic data are modelled to estimate expected mortality and mortality greater than expected is labelled as excess, but improves on the specificity of Serfling's model in that epidemic periods in the data are informed by a specific measure of influenza.

An influenza year was defined as week 26 of a given year to week 25 of the next year because the timing of influenza circulation during a given winter usually spans two calendar years (ie, extends from October of a given year to March of the next year). Mortality and laboratory data were collapsed into weekly counts for analysis. In order to differentiate excess from baseline mortality, we used laboratory data to estimate which were the epidemic weeks in the time series. We then fitted models to the mortality time series to determine excess as well as baseline mortality with reference to these epidemic weeks. Excess mortality was the sum of observed minus predicted deaths in weeks when laboratory data breached their epidemic threshold, by influenza year. Baseline trend in mortality incidence was approximated by fitting the mortality model to the death counts not labelled as excess. This model fit was then deconstructed and just the spline (ie, trend) component was plotted in order to graphically assess its shape for changes, or turning points, in the direction of the trend coincident with policy changes. In the same way, estimates of excess mortality were plotted over time to look for evidence of turning points in the trend. Estimates of excess mortality and plots of baseline trends were determined separately for the age groups 65-74, 75+ and 45-64 years (the latter age group being 'unexposed' to a change in vaccine policy or coverage over the period). Any estimates of negative excess mortality were recoded to 0 . No model was stratified by sex because an initial exploration of the data suggested similar trends by sex in the study period. A sensitivity analysis was carried out defining epidemic periods based on counts of combined laboratoryconfirmed influenza A and B virus infections (as opposed to the main analysis where this was performed using counts of influenza A infections only).

To quantify vaccine impact on excess mortality, we fitted age group-specific regression models of excess mortality for each influenza year against (1) a dummy variable having a value of 0 for 1989/1990-1999/2000 and 1 for 2000/2001-2004/2005 to capture the start of the policy targeting all those aged $65+$ years for yearly influenza vaccination (exposure of interest), (2) the minimum monthly temperature observed in each influenza year, ${ }^{29}$ (3) whether the influenza season experienced a large antigenic drift event or not $^{30}$ and (4) whether the influenza season was characterised by a mismatch between vaccine and circulating H3N2 viruses or not (from the literature). Excess deaths are right skewed and hence were transformed using $\log ($ excess +1$)$ to obtain a good approximation to normality. Models were fitted separately for the influenza A/H3N2 virus-dominated influenza seasons and for the influenza seasons dominated by other influenza virus subtypes. This was performed to allow for greater mortality, and thus potentially greater vaccine impact, in influenza seasons dominated by influenza A/H3N2 viruses than those dominated by influenza A/H1N1 or $B$ viruses. ${ }^{31}$ Vaccine mismatch was not included in models where the outcome was excess mortality in the non-influenza A/H3N2 virus-dominated seasons because both mismatch seasons were those in which influenza A/H3N2 viruses dominated. Separate sensitivity analyses were carried out (1) defining the vaccine coverage dummy variable with reference to 1998/1999 rather than 2000/2001 (because 1998/1999 was the first influenza year of age group-based targeting of vaccination (to those aged $75+$ years) ) and (2) modelling vaccination as a linear term, using derived estimates of coverage by age group in each influenza year, rather than as a dummy variable.

To establish the extent of circulation of influenza in the community over the influenza years studied, weekly rates of consultation for influenza-like illness (ILI), and for infectious and parasitic diseases (which excludes ILI), with sentinel general practices in England and Wales for 0-4 and 5-14 year-olds were plotted over time (data provided by the Royal College of General Practitioners weekly returns service). We also plotted the weekly all-age rates of laboratory-reported influenza $\mathrm{A}$ and $\mathrm{B}$ infections over time.

Models were fitted in R (R V.2.12.1 (2010-12-16), Copyright 2010 the $\mathrm{R}$ Foundation for Statistical Computing).

\section{RESULTS}

Weekly deaths registered to underlying P\&I in those aged 65 years and older in England and Wales in the period from 1975/1976 to 2004/2005 ranged from 34 deaths/ 1000000 person-weeks (in week 36 of 1984) to 481 deaths/1 000000 person-weeks (in week 7 of 1976).

\section{Excess mortality}

Excess mortality for the 65-74 years age group ranged from 0 to just over 2000 deaths per influenza year in the study period (table 1; table includes citations ${ }^{32-52}$ for the covariates whose values were drawn from the literature). For the $75+$ years age group, the range was from 0 to over 14000 deaths/influenza-year. Mortality attributable to epidemic influenza was highly variable from year to year. For neither age group does there appear to have been a turning point in the trend in excess mortality coinciding with the 2000/2001 season, when the policy of targeting all persons aged 65 years and over for yearly 
Table 1 Influenza seasons in England and Wales, 1974/1975-2004/2005: dominant variant, antigenic drift events in evoluation of influenza A/H3N2 viruses, vaccine mismatch, vaccine coverage and numbers of excess $P \& 1$ deaths by age group

\begin{tabular}{|c|c|c|c|c|c|c|c|c|c|c|}
\hline $\begin{array}{l}\text { Influenza } \\
\text { season }\end{array}$ & $\begin{array}{l}\text { Dominant } \\
\text { influenza } \\
\text { variant(s) }\end{array}$ & $\begin{array}{l}\text { Antigenic } \\
\text { drift events }^{30}\end{array}$ & $\begin{array}{l}\text { Vaccine } \\
\text { cluster* }^{*}\end{array}$ & $\begin{array}{l}\text { Minimum } \\
\text { monthly } \\
\text { temperature } \\
\left({ }^{\circ} \mathrm{C}\right)\end{array}$ & $\begin{array}{l}\text { Vaccine } \\
\text { coverage, } \\
65-74 \text { years (\%) }\end{array}$ & $\begin{array}{l}\text { Excess } \\
\text { deaths }(n), \\
65-74 \text { years }\end{array}$ & $\begin{array}{l}\text { Vaccine } \\
\text { coverage, } \\
75+\text { years (\%) }\end{array}$ & $\begin{array}{l}\text { Excess } \\
\text { deaths }(n), \\
75+\text { years }\end{array}$ & $\begin{array}{l}\text { Vaccine } \\
\text { coverage, } \\
\text { 45-64 years (\%) }\end{array}$ & $\begin{array}{l}\text { Excess } \\
\text { deaths }(n), \\
45-64 \text { years }\end{array}$ \\
\hline $1975 / 1976$ & $\mathrm{H} 3 \mathrm{~N} 2 / \mathrm{B}^{32}$ & EN72-VI75 & & 4.5 & 0 & 1910 & 0 & 9389 & 0 & 863 \\
\hline $1976 / 1977$ & $\mathrm{H} 3 \mathrm{~N}^{33}$ & & & 2 & 0 & 456 & 0 & 2986 & 0 & 203 \\
\hline $1977 / 1978$ & $\mathrm{H} 3 \mathrm{~N} 2 / \mathrm{H} 1 \mathrm{~N} 1^{32}$ & VI75-TX77 & & 2.8 & 0 & 419 & 0 & 2563 & 0 & 158 \\
\hline $1978 / 1979$ & $B^{32}$ & & & -0.4 & 0 & 14 & 0 & 25 & 0 & 0 \\
\hline $1979 / 1980$ & $\mathrm{H} 3 \mathrm{~N}^{32}$ & TX77-BA79 & & 2.3 & 0 & 0 & 0 & 95 & 0 & 0 \\
\hline $1980 / 1981$ & $\mathrm{H} 1 \mathrm{~N} 1 / \mathrm{H} 3 \mathrm{N2}^{32}$ & & & 3 & 0 & 89 & 0 & 605 & 0 & 73 \\
\hline $1981 / 1982$ & $\mathrm{~B} / \mathrm{H} 3 \mathrm{~N} 2^{34}$ & & & 0.3 & 0 & 174 & 0 & 501 & 0 & 4 \\
\hline 1982/1983 & $\mathrm{H} 3 \mathrm{~N}^{34}$ & & & 1.7 & 0 & 264 & 0 & 1947 & 0 & 48 \\
\hline $1983 / 1984$ & $\mathrm{H} 1 \mathrm{~N} 1 / \mathrm{B}^{34}$ & & & 3.3 & 0 & 88 & 0 & 742 & 0 & 38 \\
\hline $1984 / 1985$ & $\mathrm{H} 3 \mathrm{~N} 2 / \mathrm{B}^{34}$ & & & 0.8 & 0 & 74 & 0 & 395 & 0 & 5 \\
\hline $1985 / 1986$ & $\mathrm{~B}^{35}$ & & & -1.1 & 0 & 0 & 0 & 0 & 0 & 14 \\
\hline $1986 / 1987$ & $\mathrm{H} 1 \mathrm{~N} 1^{35}$ & & & 0.8 & 0 & 0 & 0 & 0 & 0 & 0 \\
\hline $1987 / 1988$ & $\mathrm{H} 3 \mathrm{~N} 2 / \mathrm{H} 1 \mathrm{~N} 1^{36}$ & BA79-SI87 & & 4.9 & 0 & 0 & 0 & 5 & 0 & 4 \\
\hline $1988 / 1989$ & $\mathrm{H} 1 \mathrm{~N} 1 / \mathrm{H} 3 \mathrm{~N}^{36}$ & & & 5.2 & 0 & 374 & 0 & 2023 & 0 & 119 \\
\hline 1989/1990 & $\mathrm{H} 3 \mathrm{~N}^{36}$ & SI87-BE89 & $\mathrm{S} 187^{37}$ & 4.9 & 22 & 2007 & 27 & 14115 & 9 & 638 \\
\hline $1990 / 1991$ & $B^{36}$ & & $B E 89^{38}$ & 1.5 & 24 & 0 & 31 & 178 & 9 & 0 \\
\hline 1991/1992 & $\mathrm{H} 3 \mathrm{~N} 2^{36}$ & & $\mathrm{BE} 89^{39}$ & 3.7 & 27 & 413 & 33 & 3302 & 10 & 111 \\
\hline $1992 / 1993$ & $\mathrm{~B} / \mathrm{H} 1 \mathrm{~N} 1^{36}$ & BE89-BE92 & $B E 89^{40}$ & 3.6 & 26 & 0 & 33 & 0 & 10 & 0 \\
\hline $1993 / 1994$ & $\mathrm{H} 3 \mathrm{~N}^{36}$ & & BE92 41 & 3.2 & 32 & 557 & 39 & 4238 & 12 & 137 \\
\hline 1994/1995 & $\mathrm{B}^{36}$ & & BE $92^{42}$ & 4.8 & 33 & 0 & 40 & 0 & 12 & 0 \\
\hline $1995 / 1996$ & $\mathrm{H} 3 \mathrm{~N}^{36}$ & BE92-WU95 & BE $92^{43}$ & 2.3 & 33 & 651 & 42 & 5365 & 12 & 226 \\
\hline $1996 / 1997$ & $\mathrm{H} 3 \mathrm{~N}^{36}$ & & WU95 ${ }^{44}$ & 2.5 & 33 & 0 & 41 & 15 & 12 & 9 \\
\hline 1997/1998 & $\mathrm{H} 3 \mathrm{~N} 2 / \mathrm{H} 1 \mathrm{~N} 1^{45}$ & WU95-SY97 & WU95 & 5.2 & 38 & 0 & 46 & 9 & 14 & 10 \\
\hline 1998/1999 & $\mathrm{H} 3 \mathrm{~N} 2 / \mathrm{B}^{46}$ & & SY $97^{46}$ & 5.3 & 37 & 628 & 49 & 6802 & 13 & 233 \\
\hline $1999 / 2000$ & H3N2 $2^{47}$ & & SY $97^{47}$ & 4.9 & 40 & 1083 & 53 & 10554 & 14 & 560 \\
\hline $2000 / 2001$ & $\mathrm{~B} / \mathrm{H} 1 \mathrm{~N} 1^{48}$ & & SY $97^{48}$ & 3.2 & $59+$ & 0 & $74 \dagger$ & 109 & 16 & 0 \\
\hline $2001 / 2002$ & $\mathrm{H} 3 \mathrm{~N} 2 / \mathrm{H} 1 \mathrm{~N} 2^{49}$ & & SY $97^{49}$ & 3.6 & $61 \dagger$ & 21 & $76 \dagger$ & 0 & 17 & 0 \\
\hline $2002 / 2003$ & $\mathrm{~B} / \mathrm{H} 3 \mathrm{~N} 2^{50}$ & SY97-FU02 & SY $97^{50}$ & 3.9 & $63+$ & 0 & $78 \dagger$ & 0 & 17 & 10 \\
\hline $2003 / 2004$ & $\mathrm{H} 3 \mathrm{~N} 2^{51}$ & & $S Y 977^{1}$ & 4.8 & $65 \dagger$ & 338 & $82 \dagger$ & 2704 & 18 & 152 \\
\hline 2004/2005 & $\mathrm{H} 3 \mathrm{~N}^{2}{ }^{52}$ & & FU02 ${ }^{52}$ & 4.3 & $64 \dagger$ & 1 & $81 \dagger$ & 27 & NA & 9 \\
\hline
\end{tabular}

*Italics indicate a mismatch between a vaccine and a dominant circulating $\mathrm{A} / \mathrm{H} 3 \mathrm{~N} 2$-virus cluster.

Estimated from coverage $65+$ years and mean ratio of coverage in the 65-74 to $65+$ years age group and the $75+$ to $65+$ years age group for $1989 / 1990-1999 / 2000$.

NA, not applicable; P\&I, pneumonia or influenza. 


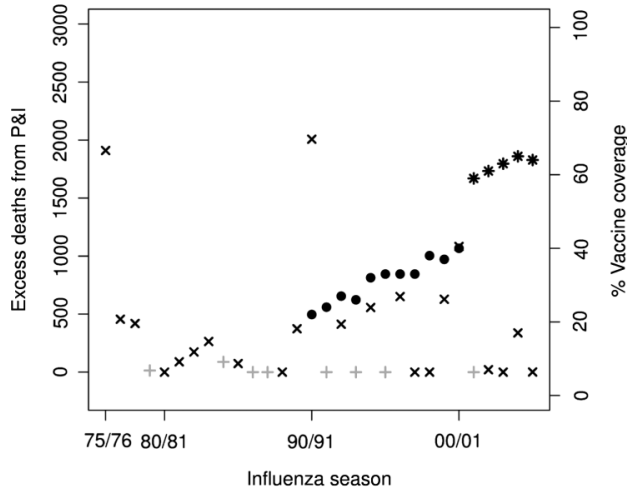

(a) $65-74$ yrs

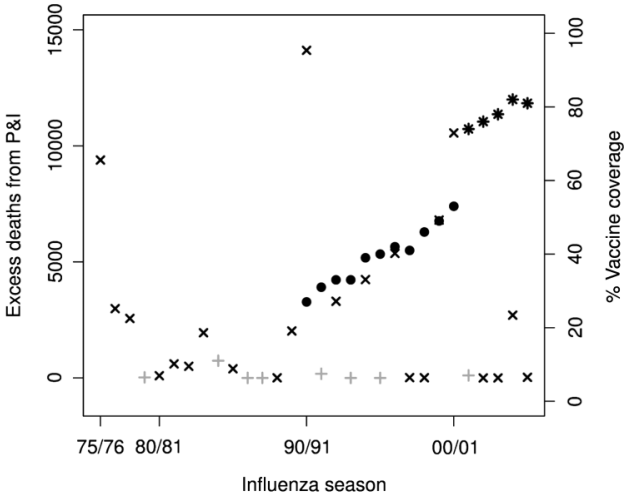

(b) $75+$ yrs

Figure 1 Excess mortality in the age groups (A) 65-74 and (B) 75+ years in each influenza season in England and Wales, stratified by the dominant influenza virus in circulation (black ' $x$ ' $=\mathrm{A} / \mathrm{H} 3 \mathrm{~N} 2$ viruses were dominant or codominant; grey ' + ' $=\mathrm{A} / \mathrm{H} 1 \mathrm{~N} 1$ or $B$ viruses were dominant). Vaccine coverage in the respective age group from published data is also shown (dots and asterisks, right axis). Asterisks indicate that vaccine coverage in these years was inferred from observed vaccine coverage in the $65+$ years age group and the average ratio of coverage in the age group $65+$ to $65-74$ years (or $75+$ years). P\&l, pneumonia or influenza.

influenza vaccination was enacted (figure 1). Similarly, there was no turning point in the trend in excess mortality in the 75+ years age group around 1998/1999 when this age group became fully targeted for yearly influenza vaccination. The direction of the trend in excess mortality over the whole time period appears to be downwards or flat. The trend in excess mortality in the 45-64 years age group is approximately flat in the same period (see online supplementary figure S1). Mean excess mortality was lower after the policy change for the age groups 6574 as well as $75+$ years, but was also lower after 2000/ 2001 than before among the 45-64 year-olds (see online supplementary table S2).

\section{Vaccine impact from multivariable regression}

The point estimates for the multiplicative effect that the policy change had on excess mortality after 2000 compared to before, adjusting for cold weather and antigenic drift in influenza A/H3N2 viruses and stratified by the dominant circulating virus subtype, are in the direction of lower excess mortality after 2000 than before for the 65-74 and 75+ years age groups, except for mortality in the seasons dominated by influenza $\mathrm{A} / \mathrm{H} 1 \mathrm{N1}$ or $\mathrm{B}$ viruses for the 75+ years age group for which the coefficient suggested higher mortality after the policy change but with a very wide CI (figure 2). Point estimates indicate a modest impact in the influenza A/H3N2 virusdominated seasons, which is unlikely to be due to chance for the $75+$ years age group, while in seasons dominated by influenza $\mathrm{A} / \mathrm{H} 1 \mathrm{~N} 1$ or $\mathrm{B}$ viruses the CIs are wide and include no effect. These findings are robust to modelling the vaccine policy change as occurring in 1998/1999 instead of 2000/2001 (data not shown). For the 45-64 years age group, the point estimates suggest lower excess mortality after 2000 than before for seasons dominated by influenza A/H3N2 viruses (figure 2). For each of the three age groups, modelling the effect of change in vaccine coverage as a linear instead of a binary (dummy) variable results in the point estimates suggesting a lower excess mortality per unit increase in vaccine coverage but with wide CIs including the null (data not shown).

\section{Trends in baseline mortality}

Long-term trends in mortality not labelled as excess, analysed as a proxy for baseline P\&I mortality, are complex, with three to four periods during which different trends were observed. For the 65-74 years age

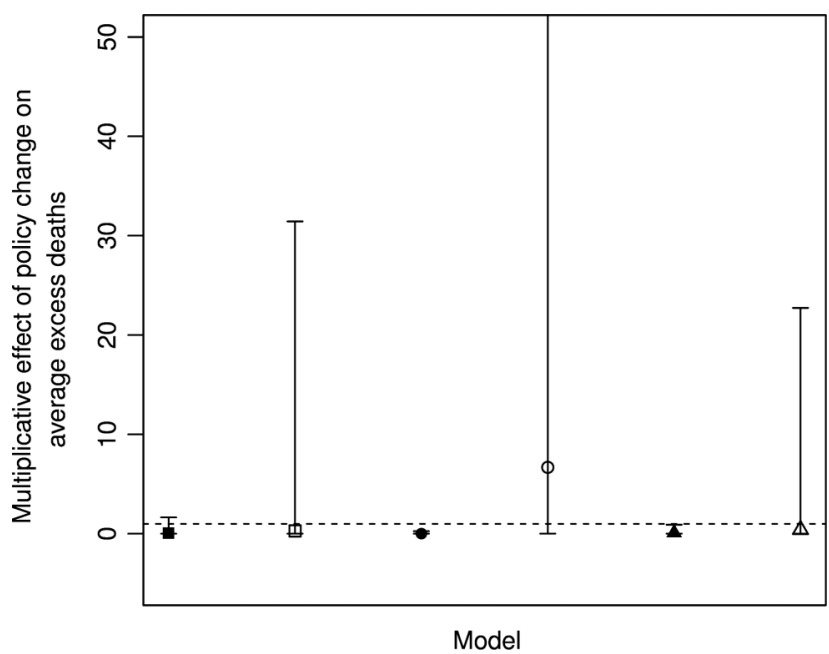

Figure 2 Summary of estimated vaccine impact from log (excess +1 ) regression models of the binary effect of the switch to age group-based targeting of yearly influenza vaccination on all those aged 65 years and older (from 2000/ 2001 onwards) compared to before 2000/2001. Coefficients are shown on the original scale and are therefore multiplicative. Squares, circles and triangles indicate the age groups 65-74, 75+ and 45-64 years, respectively. Filled symbols represent seasons dominated by influenza $A / H 3 N 2$ viruses and open symbols indicate seasons dominated by influenza $\mathrm{A} / \mathrm{H} 1 \mathrm{~N} 1$ or $\mathrm{B}$ viruses. 


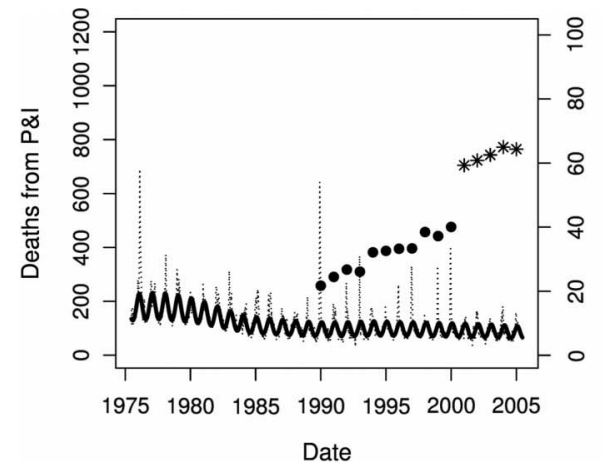

(a) $65-74$ yrs

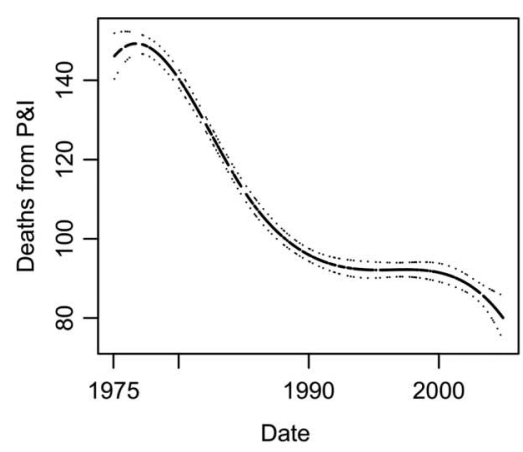

(c) $65-74$ yrs

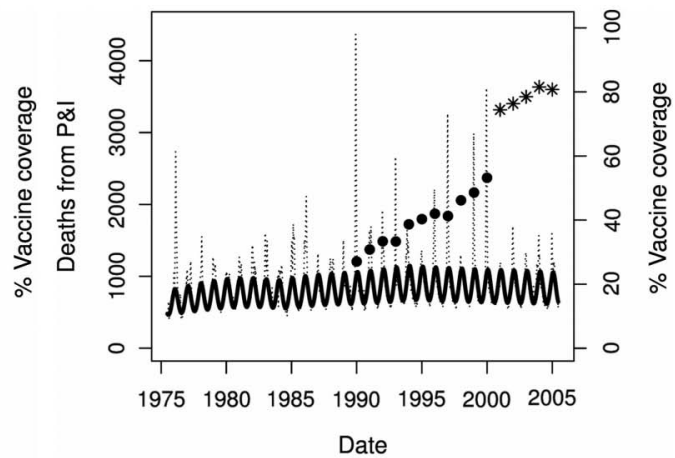

(b) $75+y r s$

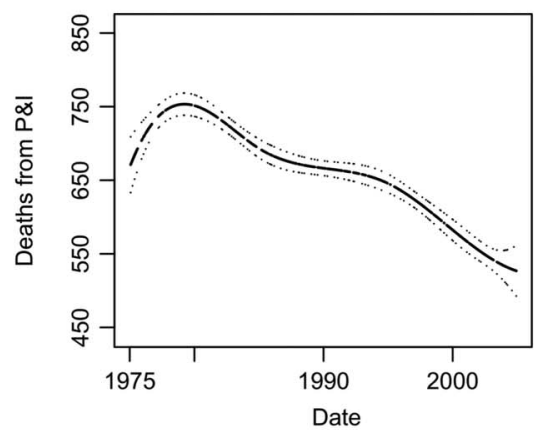

(d) $75+$ yrs

Figure 3 Model fit: the observed time series of weekly pneumonia or influenza (P\&I) deaths in the age groups (A) 65-74 years and (B) 75+ years in England and Wales between 1975 and 2005 (light dotted line on each plot). The fitted curve from the log-linear Simonsen-like model fitted excluding December to April and accounting for seasonality, long-term trend and artefacts is overlaid (dark line). Vaccine coverage (in the age groups 65-74 and 75+ years) adapted from published data is shown on the right axis of each plot (dots and asterisks). Asterisks indicate that the vaccine coverage in these years was inferred from the observed vaccine coverage in the $65+$ years age group and the average ratio of coverage in the 65-74 (or 75+) to 65+ years age group. The fitted curve can be deconstructed into its constituent parts. Thus, (C) and (D) showing the long-term trend (ie, cubic spline) component of the fitted curve (dark lines), with its $95 \% \mathrm{Cl}$ (light lines), for the $65-74$ and $75+$ years age groups, respectively. This allows a better visualisation of the shape of the long-term trend. P\&I, pneumonia or influenza.

group, rates in the study period were highest until the late 1970 s, declined until approximately 1990, plateaued until 2000 and fell after 2000 (figure 3). For the 75+ years age group, rates increased until the late 1970s, declined until the mid-1980s, plateaued until the mid-1990s and then fell. The 45-64 years age group showed a broadly similar baseline trend to that of the 65-74 years age group, though the trend was more tortuous due to smaller numbers (see online supplementary figure S2). Residuals from fitted models were normally distributed with the mean approximately 0 and SD approximately 1 with some residual autocorrelation (data not shown). Findings are robust to defining epidemic periods using laboratory reports of the influenza A infections alone or to using the influenza A and B infections for this purpose.

Secular trends in circulation of influenza in the community during the study period

During the period 2000-2005, ILI consultation rates for 0-4 and 5-14 year-olds were lower than the historical ones (see online supplementary figure S3). However, over the same time period, consultations for infectious and parasitic diseases (which excludes ILI) also appeared to decline, especially in the 5-14 years age group (see online supplementary figure S4). Rates of laboratory reported influenza infections were lower in 2000-2005 than in the previous decade (see online supplementary figure S5). Rates in 2000-2005 were similar to those observed around 1980 (figure 3).

\section{DISCUSSION}

\section{Statement of principal findings}

There is weakly supportive evidence that the switch from risk-based to age group-based targeting of influenza vaccination for older people was associated with a lower influenza-related mortality in the 4-6 years following this policy change. Results from our multivariable regression suggest that this policy change had a weak, positive impact on excess mortality in the $65-74$ as well as $75+$ years age groups. There is no indication of a downward turning point in the trend in excess mortality around 2000/2001 (when all those $65+$ years began to be targeted for vaccination) or 1998/1999 (when those 75+ years began to be targeted). There were fewer excess 
deaths, on average, in the seasons after the policy change than before (including in the 45-64 years age group), which argues against a strong, specific impact of the policy change on excess mortality. Trends in baseline, as opposed to excess, mortality do show a suggestion of a downward turning point in the mid-1990s for the 75+ years age group and around 2000 for the 65-74 years age group, coinciding approximately with the timing of the changes to specifically target these age groups for vaccination. Results obtained by fitting the various models to data for the 45-64 years age group, in whom vaccine coverage was largely static over the study period, are in a similar direction but are of a smaller magnitude than the apparent impact in the 65-74 and $75+$ years age groups, suggesting that there may have been other factors acting to bring down P\&I mortality at the time of the policy changes. Thus, our analysis is consistent with a small mortality impact in the 65-74 and $75+$ years age groups during the 6 years after policies to wholly target these age groups for yearly influenza vaccination were enacted.

\section{Strengths and weaknesses of the study}

There are a number of strengths to the work undertaken, as well as several limitations. The key strengths are that we estimated excess mortality and long-term trend in mortality over a long period (30 years). We carefully controlled for changes to death coding and laboratory practices which occurred over this time period. The analytical approach we used, which is similar to the one used by many others, ${ }^{15} 16$ of modelling seasonality and trend using splines in addition to sinusoidal terms (full details provided in online supplementary material), is a highly flexible method of fitting complex patterns and trends that is especially helpful when modelling a long time series. The outcome we chose to model (deaths from underlying P\&I) is the most specific option available which allows sufficient numbers of deaths for analysis. This choice maximised our ability to discern high mortality impact influenza years from those less so, and thus to detect vaccine impact. Analysing underlying P\&I of course means that our estimates of excess mortality underestimate the burden of mortality due to all respiratory disease (which includes bronchitis), cardiovascular disease and other causes of death which may be linked to influenza. ${ }^{19}{ }^{20}$ However, it was not the aim of this work to estimate the total mortality burden due to influenza. Further adding to the specificity of our outcome was our designation of epidemic periods in the mortality data with reference to the time series of laboratoryconfirmed influenza A infections. A still better approach might have been to use influenza A/H3N2 infections, the subtype most often associated with influenza years when there is substantial mortality, ${ }^{53}$ but the laboratory data available for this analysis were not broken down by subtype. The limitations of our work include the following: that our estimate of the extent of variability in excess mortality across influenza years is likely to be an underestimate because our model failed to explain all variability in the mortality data (as evidenced by a small amount of autocorrelation in residuals, data not shown). This may be because we did not include temperature or other climatic variables in the models estimating excess mortality. We did adjust for temperature in multivariable regression models of vaccine impact on excess mortality and, as such, our analysis of vaccine impact should not be confounded by temperature. However, our results regarding long-term trends in non-excess mortality may be confounded by temperature as there is evidence that minimum winter temperatures have increased since the early 1960 s. ${ }^{54}$ In using laboratory data to inform epidemic periods in the mortality data, we made no allowance for a lag between the increase, or peak, in incidence of laboratory-reported infections and the timing of excess deaths associated with these infections. This is likely to have led us to underestimate excess mortality, assuming that the peak in deaths rarely precedes and generally coincides with or follows the peak in laboratory reports. However, the rise in influenza activity in the community in terms of general practitioner (GP) consultations generally coincides with the rise in numbers of laboratory reports of influenza infections, ${ }^{36}$ and, during influenza years dominated by circulation of influenza A viruses, peak weekly GP consultation rates for ILI in the $45-64,65-74$ and $\geq 75$ years age groups tend to coincide with same age peaks in respiratory deaths, plus or minus 1 week (eg, 1995/1996, 1996/ 1997, 1998/1999 and 1999/2000). ${ }^{18}$ Hence, the potential bias in our estimates of excess mortality is likely to be minimal because the weeks we might have missed by not allowing for a lag will be close to the start or end of the period of influenza circulation and thus will make up a small proportion of the total influenza attributable P\&I deaths in a given winter. Our estimates of long-term trends may be confounded by the trends in comorbidities linked to smoking or by the long-term patterns in other comorbidities associated with respiratory mortality, which we have not accounted for. While it would have been possible to test for a change in slope of the longterm trend with a piecewise linear approximation, we did not do this because issues of confounding by these types of time-varying covariates, which we are only able to speculate about, would limit the interpretability of any coefficient. Finally, the separate vaccine coverage estimates that we present for the 65-74 and 75+ years age groups from 2000/2001 onwards are sensitive to our assumption of a constant ratio of vaccine coverage in the 65-74 and 75+ years age groups from 2000/2001 onwards (described in online supplementary file). The assumption of a constant ratio is unlikely to be true and, as such, we have probably underestimated coverage in the 65-74 years age group and overestimated it in the $75+$ years age group; the increase in coverage in 2000/ 2001 was probably disproportionately accounted for by an increase in coverage in the 65-74 years age group, newly targeted as fully 'at risk' from the 2000/2001 
influenza season. Our main findings are unaffected by this because we focus on the results from regression models of the effect of change in vaccination policy as a binary variable.

\section{Strengths and weaknesses in relation to other studies, discussing important differences in results}

Using as a reference the previous estimates which exist of excess respiratory mortality in England and Wales, our estimates appear to be a valid reflection of variability between years and between age groups in influenza-attributable mortality. Findings as to the vaccine impact from other settings have been mixed; thus, our observation of perhaps some limited impact is not inconsistent with reports from other settings which analysed the impact of similar levels of vaccine coverage to that achieved in the UK. Previous estimates of excess all-age respiratory mortality for the influenza years 1975/1976, 1976/1977 and 1977/1978 are higher than our excess P\&I estimates for these years, but the relative magnitude of the excess mortality between these years matches our estimates well (see online supplementary table S1). The absolute value of the previous estimates is higher than ours because the authors included bronchitis deaths and analysed all ages combined, not just the elderly. For the influenza years 1994/1995-1999/ 2000, published estimates of average influenzaattributable respiratory mortality by age group (45-64, 65-74 and 75+ years) are higher than our averages for the same age groups over the same period, but ratios between different age groups are similar to our estimates. Differences in the magnitude of estimates from the two methods will be not only because they include bronchitis deaths but also because the rate differencetype method used by Fleming and colleagues tends to produce higher estimates of excess than the Serfling-type method we used, due to a lower reference mortality and the mortality rate in the 'influenza-active periods' being entirely attributed to influenza. ${ }^{55}$ There has been much interest in measuring the impact of influenza vaccination campaigns in other settings. ${ }^{14} 151756$ Excess all-cause mortality declined in the Dutch elderly after the introduction of universal yearly vaccination of those aged 65 years and over, which saw yearly coverage reach $80 \%{ }^{14}$ Excess all-cause mortality and influenzarelated hospitalisations and GP visits declined more in Ontario than in other Canadian provinces after the introduction of universal yearly vaccination for all Ontario residents. ${ }^{56}$ Analyses of the impact of rising vaccine coverage of the elderly on excess all-cause mortality in the USA, Italy and Catalonia did not detect impact perhaps because, in the case of the USA and Italy, the coverage did not reach high enough levels for long enough during the study period, or because, as in Catalonia, there were limited data available, prior to coverage exceeding $60 \%$, to provide a baseline against which to estimate impact. ${ }^{15-17}$ Because the ratios of our estimates of excess P\&I between adjacent seasons, and between age groups, are consistent with previous work using different models, we are confident that our impression of the relative magnitude of P\&I mortality between age groups and from one influenza year to the next year are a true reflection of patterns in influenza mortality during the study period. Our observation of some evidence for vaccine impact on excess mortality in the 6 years after implementation of age group-based influenza vaccination is consistent with the findings from other temperate northern hemisphere settings which have attained similar levels of vaccine coverage in the elderly, where some studies have demonstrated an impact and others have not.

\section{Meaning of the study: possible explanations and implications for clinicians and policymakers}

Our analysis suggests that high yearly vaccination coverage of the elderly had a small impact on P\&I deaths in the 4-6 years following implementation of age groupbased targeting of the elderly for influenza vaccination. Alternative explanations are that there was less influenza around during the time of high vaccine coverage, making it look like vaccination produced an impact on mortality when it did not. The fact that we observed smaller magnitude but same direction associations between the timing of policy changes in the elderly and mortality in the 45-64 years age group, in whom vaccination policy and coverage were approximately constant over the study period, supports this alternative explanation. However, our analysis of the extent to which influenza circulated in the community in the study period does not provide clear answers: consultations among children for both ILI as well as infectious and parasitic diseases (which excludes respiratory disease) were lower in the 2000-2005 period than earlier in the study period. It is difficult to interpret lower ILI rates as strong evidence for less influenza circulating in 2000-2005 than previously since the decline in rates of infectious and parasitic diseases rates then still needs explaining. It may in part be that there was progressively lower use of GP services in the 2000-2005 period. Rates of laboratory-reported influenza infections for all ages reported to HPA CfI were certainly lower in 2000-2005 than in the previous decade, and were similar to the rates observed around 1980 when excess mortality also appeared to be low for several consecutive years. It is difficult to interpret the long-term trends in laboratory reports of influenza infections because of the changing testing practices and changing volumes of test requisitions over the time period. For example, between 1975 and 1992, the number of laboratory reports of viral infections doubled ${ }^{57}$; this is unlikely to reflect a doubling of viral infections over this period. If more tests were requisitioned in 2000-2005 than in the years around 1980, similar rates of positive reports in the two periods would suggest less viral activity in 2000-2005 than around 1980. However, it is hard to see how less influenza in circulation later than earlier in the study 
period could explain a turning point in baseline mortality in the 65-74 and 75+ years age groups approximately coincident with policy changes to specifically target these age groups for vaccination. We think the observation of a turning point in baseline mortality reflects partly a non-specific impact of influenza vaccination on respiratory mortality in the elderly that is not directly attributable to influenza as well as partly a specific impact of influenza vaccination given that analysing excess mortality necessarily means some truly influenza-attributable mortality (ie, that which does not breach the epidemic threshold) contributes to baseline. A further possible alternative explanation for the apparent, small influenza vaccine impact we have observed is that this is really an impact of pneumococcal vaccination. This is unlikely to be true; to the end of the 2004/2005 influenza year, less than $30 \%$ of people aged 65 years and over had received the recommended 23-valent pneumococcal polysaccharide vaccine. ${ }^{58}$ Pneumococcal conjugate vaccination of infants and children, which might be expected to provide indirect protection to the elderly, has only been routinely recommended since 2006. It is possible that there is some other factor which also changed over the time period and which explains part of the mortality impact we observed (eg, trends in comorbidities linked to smoking).

\section{Unanswered questions and future research}

Influenza vaccine impact can be difficult to measure due to its reliance on surveillance data which are generally not collected in a consistent way over time, and because of the variable nature of influenza activity over time. One way of adding to the evidence base is to look at vaccine effectiveness. In order to strengthen the evidence base for influenza vaccine effectiveness in the elderly, further good-quality cohort and case-control studies across multiple influenza seasons with varying degrees of match between vaccine and circulating variants, as well as adequate control for negative confounding by indication (sicker elderly being preferentially offered vaccination) and positive confounding by healthier older people putting themselves forward for vaccination, are required. It remains unclear to what extent our findings are due to there being less influenza around 2000-2005 than before this time or to confounding by factors such as trends in comorbidities linked to smoking. Analyses including recent influenza seasons, which experienced a pandemic $(2009 / 2010)$ and a severe influenza season dominated by the H1N1pdm09 virus $(2010 / 2011)$ and which saw the vaccine uptake of the elderly further increase, will help to answer these questions and to monitor whether the modest mortality impact we observed in the 6 years after age group-based targeting of vaccination began has been sustained. This analysis will be complicated by the presence of pneumococcal vaccination of infants and the elderly, making teasing apart the effects of how much influenza circulated during the time period, the impact of influenza vaccination and the impact of pneumococcal vaccination a challenge. A way of addressing this would be to calculate the attack rates based on serological data now being collected by HPA CfI.

\section{Author affiliations}

${ }^{1}$ Department of Infectious Disease Epidemiology, Faculty of Epidemiology \& Population Health, London School of Hygiene \& Tropical Medicine, London, UK

${ }^{2}$ Department of Zoology, University of Cambridge, Cambridge, UK

${ }^{3}$ Fogarty International Center, National Institutes of Health, Bethesda

Maryland, USA

${ }^{4}$ Department of Non-Communicable Disease Epidemiology, Faculty of Epidemiology \& Population Health, London School of Hygiene \& Tropical Medicine, London, UK

${ }^{5}$ Statistical Platforms and Technologies, GlaxoSmithKline, Medicines Research Centre, Stevenage, UK

Acknowledgements The authors are very grateful to the reviewers whose comments greatly improved this manuscript. Pneumonia or influenza (P\&l) data were provided by Cleo Rooney and Emma Gordon from the Office for National Statistics. Laboratory data were provided by Carol Joseph and Joy Field of the Health Protection Agency Centre for Infections. General practitioner consultation data were provided by Douglas Fleming and Alex Elliot of the Royal College of General Practitioners.

Contributors AGM, PM and JCW conceived the study. AGM is the guarantor and conducted the analysis, led the interpretation and wrote the manuscript. JCW, PM and CAR contributed to the interpretation and subsequent drafts of the manuscript. All authors approved the final version of the manuscript to be published.

Funding This work was supported by a Researcher Development Award to AGM from the National Institute of Health Research, UK (fellowship grant number RDA06/068).

Competing interests JCW is currently employed by, and holds stock options with, GlaxoSmithKline (GSK), but the relationship of JCW to GSK had no influence on the work or on the decision to publish it.

Ethics approval This study was approved by the ethics committee of the London School of Hygiene \& Tropical Medicine (approval number 5109).

Provenance and peer review Not commissioned; externally peer reviewed.

Data sharing statement No additional data are available.

Open Access This is an Open Access article distributed in accordance with the Creative Commons Attribution Non Commercial (CC BY-NC 3.0) license, which permits others to distribute, remix, adapt, build upon this work noncommercially, and license their derivative works on different terms, provided the original work is properly cited and the use is non-commercial. See: http:// creativecommons.org/licenses/by-nc/3.0/

\section{REFERENCES}

1. Department of Health: from the Chief Medical Officer, the Chief Nursing Officer and the Chief Pharmaceutical Officer. Influenza immunisation. PL/CMO/2000/3. London: Department of Health, 2000.

2. Joseph C, Goddard N, Gelb D. Influenza vaccine uptake and distribution in England and Wales using data from the General Practice Research Database, 1989/90-2003/04. J Public Health (Oxf) 2005;27:371-7.

3. Butt S, Zhang N, Joseph CA. Vaccination uptake among the 65 years and over and under 65 years at risk in England 2006-07. London: Health Protection Agency Centre for Infections, 2007.

4. Begum F, Pebody R. Seasonal influenza vaccine uptake amongst GP patient groups in England. London: Department of Health, 2012.

5. Fedson DS, Nichol KL. Influenza vaccination: policy versus evidence: no gap between policy and evidence. BMJ 2006;333:1020.

6. Jefferson T. Influenza vaccination: policy versus evidence. BMJ 2006;333:912-15. 
7. Mangtani $P$, Hall AJ, Armstrong BG. Influenza vaccination: the case for a gap in the evidence is flawed. BMJ 2006;333.

8. Simonsen L, Viboud C, Taylor R. Influenza vaccination in elderly people. Lancet 2005;366:2086.

9. Jefferson T, Di Pietrantonj C, Al-Ansary LA, et al. Vaccines for preventing influenza in the elderly. Cochrane Database Syst Rev 2010(2):CD004876

10. Osterholm MT, Kelley NS, Sommer A, et al. Efficacy and effectiveness of influenza vaccines: a systematic review and meta-analysis. Lancet Infect Dis 2012;12:36-44.

11. Govaert TM, Thijs CT, Masurel N, et al. The efficacy of influenza vaccination in elderly individuals. A randomized double-blind placebo-controlled trial. JAMA 1994;272:1661-5.

12. Mangtani $\mathrm{P}$, Cumberland $\mathrm{P}$, Hodgson $\mathrm{CR}$, et al. A cohort study of the effectiveness of influenza vaccine in older people, performed using the United Kingdom general practice research database. $J$ Infect Dis 2004;190:1-10.

13. Ortqvist A, Granath F, Askling J, et al. Influenza vaccination and mortality: prospective cohort study of the elderly in a large geographical area. Eur Respir J 2007;30:414-22.

14. Jansen AGSC, Sanders EAM, Nichol KL, et al. Decline in influenza-associated mortality among Dutch elderly following the introduction of a nationwide vaccination program. Vaccine 2008;26:5567-74.

15. Simonsen L, Reichert TA, Viboud C, et al. Impact of influenza vaccination on seasonal mortality in the US elderly population. Arch Intern Med 2005;165:265-72.

16. Rizzo C, Viboud C, Montomoli E, et al. Influenza-related mortality in the Italian elderly: no decline associated with increasing vaccination coverage. Vaccine 2006;24:6468-75.

17. Muñoz MP, Soldevila N, Martínez A, et al. Influenza vaccine coverage, influenza-associated morbidity and all-cause mortality in Catalonia (Spain). Vaccine 2011;29:5047-52.

18. Fleming DM, Cross KW, Pannell RS. Influenza and its relationship to circulatory disorders. Epidemiol Infect 2005;133:255-62.

19. Hardelid P, Pebody R, Andrews N. Mortality caused by influenza and respiratory syncytial virus by age group in England and Wales 1999-2010. Influenza Other Respi Viruses 2013;7:35-45.

20. Pitman RJ, Melegaro A, Gelb D, et al. Assessing the burden of influenza and other respiratory infections in England and Wales. $J$ Infect 2007:54:530-8.

21. Serfling RE. Methods of current statistical analysis of excess pneumonia-influenza death. Public Health Rep 1963;78:494-506.

22. Fleming D, Wood M. The clinical diagnosis of influenza. Curr Med Res Opin 2002;18:338-41.

23. Barker WH, Mullooly JP. Underestimation of the role of pneumonia and influenza in causing excess mortality. Am J Public Health 1981;71:643-5.

24. Housworth J, Langmuir AD. Excess mortality from epidemic influenza, 1957-1966. Am J Epidemiol 1974;100:40-8.

25. Janssen $F$, Kunst AE. ICD coding changes and discontinuities in trends in cause-specific mortality in six European countries, 1950-99. Bull World Health Organ 2004;82:904-13.

26. Devis T, Rooney C. Death certification and the epidemiologist. Health Stat Q 1999;01:21-33.

27. Rooney C, Griffiths C, Cook L. The implementation of ICD-10 for cause of death coding - some preliminary results from the bridge coding study. Health Stat Q 2002;13:31-41.

28. Brock A, Griffiths C, Rooney C. The impact of introducing ICD-10 on analysis of respiratory mortality trends in England and Wales. Health Stat $Q$ 2006;29:9-17.

29. Met Office Hadley Centre Central England Temperature Data: Monthly HadCET mean [database on the Internet]. http://www. metoffice.gov.uk/research/hadleycentre/CR_data/Daily/HadCET_act. txt (accessed 18 Jun 2008).

30. Smith DJ, Lapedes AS, De Jong JC, et al. Mapping the antigenic and genetic evolution of influenza virus. Science 2004;305:371-6.

31. Fleming DM. The contribution of influenza to combined acute respiratory infections, hospital admissions, and deaths in winter. Commun Dis Public Health 2000;3:32-8.

32. Pereira MS, Chakraverty P. Influenza in the United Kingdom 1977-1981. J Hyg (Lond) 1982;88:501-12.

33. Pereira M, Assaad FA, Delon PJ. Influenza surveillance. Bull World Health Organ 1978;56:192-203.
34. Chakraverty $P$, Cunningham P, Shen GZ, et al. Influenza in the United Kingdom 1982-85. J Hyg (Lond) 1986;97:347-58.

35. Smith DJ, Forrest $\mathrm{S}$, Ackley $\mathrm{DH}$, et al. Variable efficacy of repeated annual influenza vaccination. Proc Natl Acad Sci USA 1999;96:14001-6.

36. Fleming DM, Zambon M, Bartelds Al, et al. The duration and magnitude of influenza epidemics: a study of surveillance data from sentinel general practices in England, Wales and the Netherlands. Eur J Epidemiol 1999;15:467-73.

37. Recommended composition of influenza virus vaccines for use in the 1989-1990 season. Wkly Epidemiol Rec 1989;64:53-60

38. Recommended composition of influenza virus vaccines for use in the 1990-1991 season. Wkly Epidemiol Rec 1990;65:53-6.

39. Joseph CA, Dedman D, Fern K, et al. Influenza surveillance in England and Wales: November 1991-June 1992. Commun Dis Rep CDR Rev 1992;2:R149-52.

40. Dedman D, Joseph CA, Chakraverty P, et al. Influenza surveillance, England and Wales: October 1992-June 1993. Commun Dis Rep CDR Rev 1993;3:R184-6.

41. Dedman DJ, Joseph CA, Chakraverty $P$, et al. Influenza surveillance, England and Wales: October 1993 to June 1994. Commun Dis Rep CDR Rev 1994;4:R164-8.

42. Hutchinson EJ, Joseph CA, Chakraverty $P$, et al. Influenza surveillance in England and Wales: October 1994 to June 1995. Commun Dis Rep CDR Rev 1995:5:R200-4.

43. Hutchinson EJ, Joseph CA, Zambon M, et al. Influenza surveillance in England and Wales: October 1995 to June 1996. Commun Dis Rep CDR Rev 1996;6:R163-9.

44. Dedman DJ, Joseph CA, Zambon M, et al. Influenza surveillance in England and Wales: October 1996 to June 1997. Commun Dis Rep CDR Rev 1997;7:R212-19.

45. Dedman DJ, Zambon M, Buynder PV, et al. Influenza surveillance in England and Wales: October 1997 to June 1998. Commun Dis Public Health 1998:1:244-51.

46. Whiting $\mathrm{P}$, Joseph CA, Zambon M, et al. Influenza activity in England and Wales: October 1998 to June 1999. Commun Dis Public Health 1999;2:273-9.

47. Goddard NL, Joseph CA, Zambon M, et al. Influenza surveillance in England and Wales: October 1999 to May 2000. Commun Dis Public Health 2000;3:261-6.

48. Goddard NL, Joseph CA, Zambon M, et al. Influenza surveillance in the United Kingdom: October 2000 to May 2001. Commun Dis Rep CDR Suppl 2001:1-7

49. Crofts JP, Goddard NL, Joseph CA, et al. Influenza surveillance in the United Kingdom: October 2001 to May 2002. Commun Dis Rep CDR Suppl 2002:1-7.

50. Crofts JP, Joseph CA, Zambon M, et al. Influenza surveillance in the United Kingdom: October 2002 to May 2003. Commun Dis Rep CDR Suppl 2004;14:1-9.

51. Cooke MK, Crofts JP, Joseph CA, et al. Influenza and other respiratory viruses surveillance in the United Kingdom: October 2003 to May 2004. Commun Dis Rep CDR Suppl 2005;15:1-8.

52. Zhao H, Cooke MK, Joseph CA, et al. Surveillance of influenza and other respiratory viruses in the United Kingdom: October 2004 to May 2005. Commun Dis Rep CDR Suppl 2006;16:1-8.

53. Thompson WW, Shay DK, Weintraub E, et al. Mortality associated with influenza and respiratory syncytial virus in the United States. JAMA 2003;289:179-86.

54. Perry M. Climate memorandum no 21: a spatial analysis of trends in the UK climate since 1914 using gridded datasets. London: Met Office, 2006

55. Thompson WW, Weintraub E, Dhankhar P, et al. Estimates of US influenza-associated deaths made using four different methods. Influenza Other Respi Viruses 2009;3:37-49.

56. Kwong JC, Stukel TA, Lim J, et al. The effect of universal influenza immunization on mortality and health care use. PLoS Med 2008:5: e211.

57. Grant AD, Eke B. Application of information technology to the laboratory reporting of communicable disease in England and Wales. Commun Dis Rep CDR Rev 1993;3:R75-8.

58. Health Protection Agency Centre for Infections. Pneumococcal Vaccination Uptake Monitoring on behalf of the Department of Health. http://www.hpa.org.uk/Topics/InfectiousDiseases/ InfectionsAZ/Pneumococcal/PPVUptake/ (accessed 18 Sep 2012). 